\title{
A Model Study on the Particle Dispersion and Fluid-Particle Interaction in Slurry of Liquid Alloy and Ceramic Particle
}

\author{
By P. K. Ghosh*† and S. Ray*
}

\begin{abstract}
Cold model experiments have been conducted with water and poorly wetting plastic beads to visually observe the role of stirring speed and the size of the impeller on the retention of beads in water. It has been observed that the exposure of the impeller surface from water initiates the incorporation of beads in water and the extent of incorporation is enhanced with increasing exposure of the impeller. The exposure of the impeller starts at a higher speed of stirring for an impeller of a smaller diameter. It has also been noticed that a deviation of these mixing parameters from the optimum level cause either the reduced number of immersed particles or the rejection of the beads and sometimes even the accumulation of the beads at the bottom of the impeller and thereby reduces the extent of incorporation of particles.
\end{abstract}

(Received June 8, 1987)

Keywords: cold model experiment, mixing, impeller size, stirring speed, retention of plastic beads in water

\section{Introduction}

Fabrication of metal base particulate composite by foundry methods has been pursued $^{(1)-(3)}$ over a decade. The major problems encountered in production of particulate composites by these methods are the retention of non-metallic particles in molten metals or alloys due to their poor wettability and homogeneous distribution of the particles in the cast composites. Under favourable conditions of fluid motion a longer time of holding of ceramic particles inside a vigorously agitated molten alloy may improve the wetting and the distribution of the particles. In earlier studies $^{(4)(5)}$ on short fiber composites the maintenance of suitable mixing conditions has been found important for incorporation of particles or short fibers in the composites. In this regard the mixing parameters such as the stirring speed, the size and the position of the impeller inside the melt have been identified ${ }^{(4)}$ to play a significant role. In the particulate com-

* Department of Metallurgical Engineering, University of Roorkee, Roorkee-247667, India.

$\dagger$ Presently in Department of Mechanical and Industrial Engg., University of Roorkee. posite produced by the vortex method the increase in particle retention has been found ${ }^{(4)}$ to enhance the porosity content of the composite linearly with a significant coefficient of correlation. This behaviour indicates a definite role of particles in the introduction of air bubbles inside the melt during their incorporation. The presence of porosity in particulate composite produced by the foundary method has been found to influence significantly its mechanical properties at ambient ${ }^{(6)(7)}$ and elevated temperatures. ${ }^{(8)}$ Thus, it is essential to control the level of porosity in the cast particulate composite. However, in the earlier studies ${ }^{(4)(5)}$ the influence of the mixing parameters on the particle-fluid interaction inside the melt and particle-air bubble configuration at the vortex has been little understood.

In the present investigation model experiments have been conducted with water and poorly wetting plastic beads to gain a qualitative understanding from the visual observation of the role of stirring speed and the size of the impeller on the movement of particles and air bubbles inside the liquid. This study has been undertaken to understand the effect of these mixing parameters in controlling the particle retention and its distribution along with the porosity content in the cast metal com- 
posite fabricated by the vortex method.

\section{Experimental}

The plastic beads of about $2.5 \mathrm{~mm}$ diameter in average size with poor wettability and density lower than water has been chosen to carry out the experiments. The water as a fluid medium has been taken in a flat bottom transparent glass beaker of internal diameter, $D=75 \mathrm{~mm}$. The flat $3 \mathrm{~mm}$ thick turbine type four-blade impeller (Fig. 1) of three different diameters $(d)$ having $d / D$ ratios of $0.48,0.66$ and 0.82 are used for stirring water. The stirrer having the impeller fixed with its shaft is vertically placed at the centre of the liquid. During all the experiments the height of water inside the beaker $(H)$ with the stirrer in it and the position of the impeller inside water have been so maintained that $h / H=0.15$ where $h$ is the height of the impeller from the bottom of the beaker. The speed of the stirrer is regulated with the help of an auto-transformer and the stirring speed is measured by using a stroboscope.

Plastic beads weighing about $5 \mathrm{~g}$ are added on the surface of the water at rest ensuring that

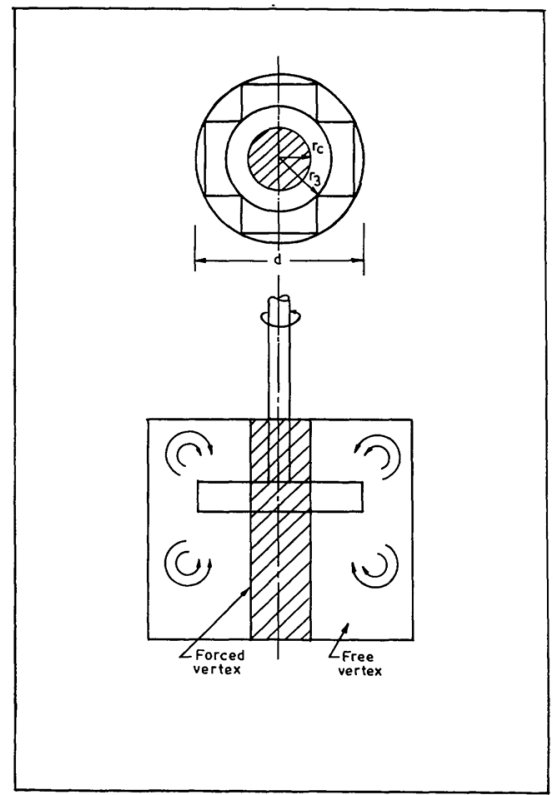

Fig. 1 Schematic diagram of the impeller and simplified flow model of a concentrically agitated mixing system. they are floating on it. The stirring speed is raised slowly by a gradual increase in the power input to the stirrer. The same procedure is followed during the experiments carried out by using different size of impeller. The vortex formation and the movement of plastic beads in water during stirring are observed. During the experiments carried out with impellers of three different sizes the stirring speed at which the plastic beads start entering the water is noted visually. The behaviour of particles in water has been photographed with the help of a movie camera at a speed of 25 photographs per second.

\section{Results}

The effects of stirring speed on the distribution of plastic beads in water are shown in Figs. 2, 3 and 4, where the stirring has been carried out with impellers of different sizes having $d / D$ of $0.82,0.66$ and 0.48 . Figure 2 shows that during stirring with the impeller of $d / D=0.82$ at a slow speed the beads have been mostly retained on the surface of the vortex. At this speed of stirring a few beads are visible inside the water due to a higher kinetic energy imparted during the addition. A large scale entry of the beads inside water has initiated only when the stirring speed reaches an optimum value exposing the impeller surface. With an increase in stirring speed the bottom of the vortex surface comes down and touches the impeller surface to expose it. A further increase in stirring speed has increased the incorporation of beads. However, at a very high speed when a substantial part of the impeller surface has been exposed the incorporated beads are found to accumulate just at the bottom of the impeller minimising the amount of incorporation of the beads in the liquid medium.

In other two experiments carried out with impellers of $d / D$ ratios of 0.66 and 0.48 the same behaviour vis-a-vis incorporation of beads as reported above, has been observed as shown in Figs. 3 and 4, respectively. However, at higher speeds of stirring with the impeller of $d / D=0.66$ it has been observed that after achieving the maximum incorporation of beads a further increase in stirring speed 


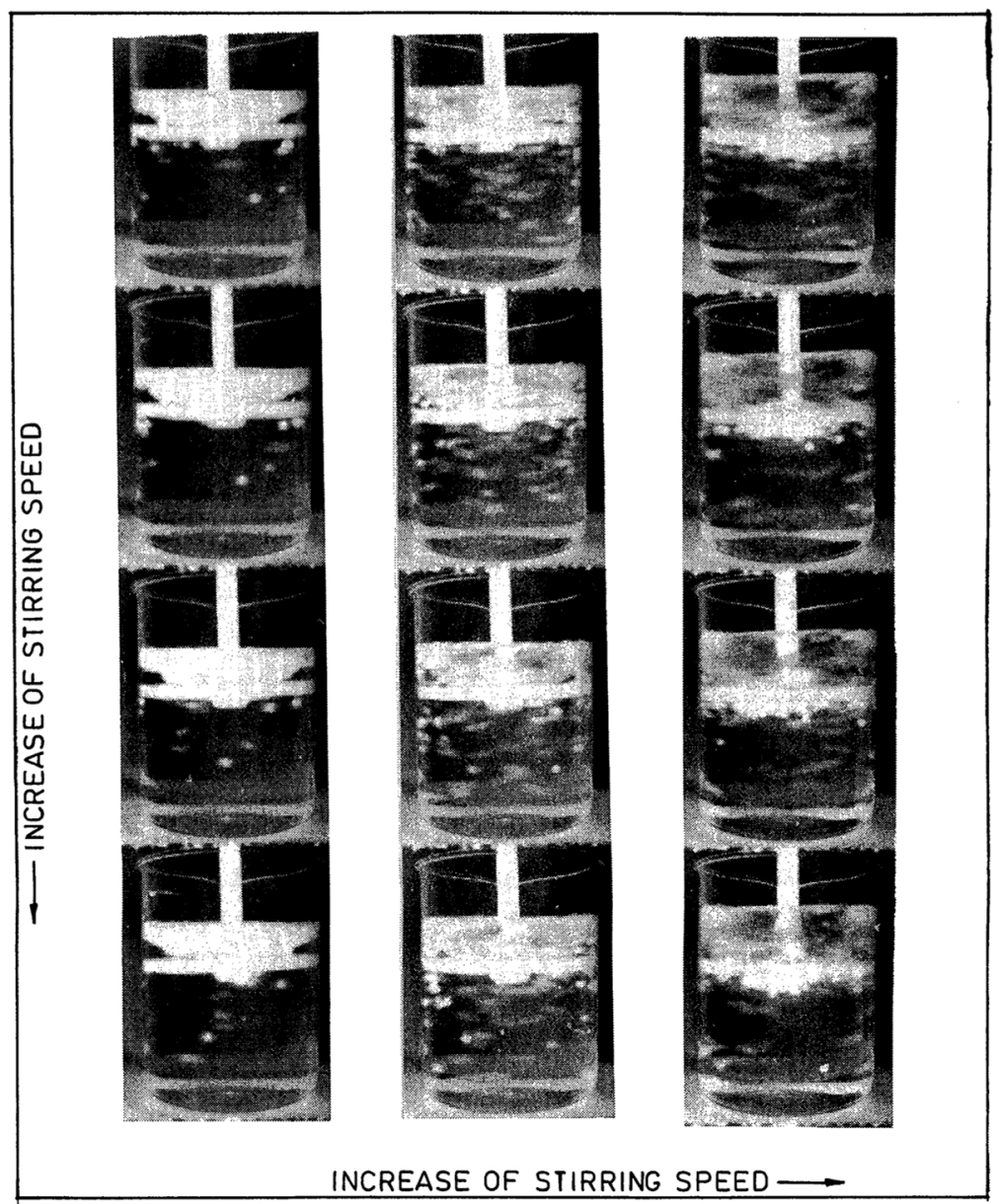

Fig. 2 Photographs showing the effect of stirring speed on the incorporation of plastic beads in water during the cold model experiment with an impeller of $d / D=0.82$.

results in pushing the vortex surface below the impeller. The beads have been found to be attracted to and accumulated at this vortex surface below the impeller making the bulk of water free from the beads as shown in Fig. 3. The extent of the retention and distribution of the beads inside water is limited when the stirring has been carried out by the impeller of $d / D=0.48$. Figure 4 shows that even at a stirring speed at which the beads start entering water leaving the vortex, there is a tendency for the beads to move around the wall of the container near the top surface of the liquid and join the vortex surface eventually without being distributed in the medium. At a higher speed of stirring the beads have been found to come down towards the bottom of the container, but they show a tendency to move at the side of the conatainer wall without being distributed throughout the liquid medium. However, with a further increase in stirring speed the turbulence at the surface of the liquid has become severer. As such the liquid motion has been found to be interrupted and the beads have been found again to join the vortex at the side of the container wall.

The initiation of exposure of the impeller during stirring has been found to occur at a different stirring speed with a change in $d / D$ ratio of the impeller. It may be noted that at this stirring speed a sudden incorporation of the beads in water has been observed. The cor- 


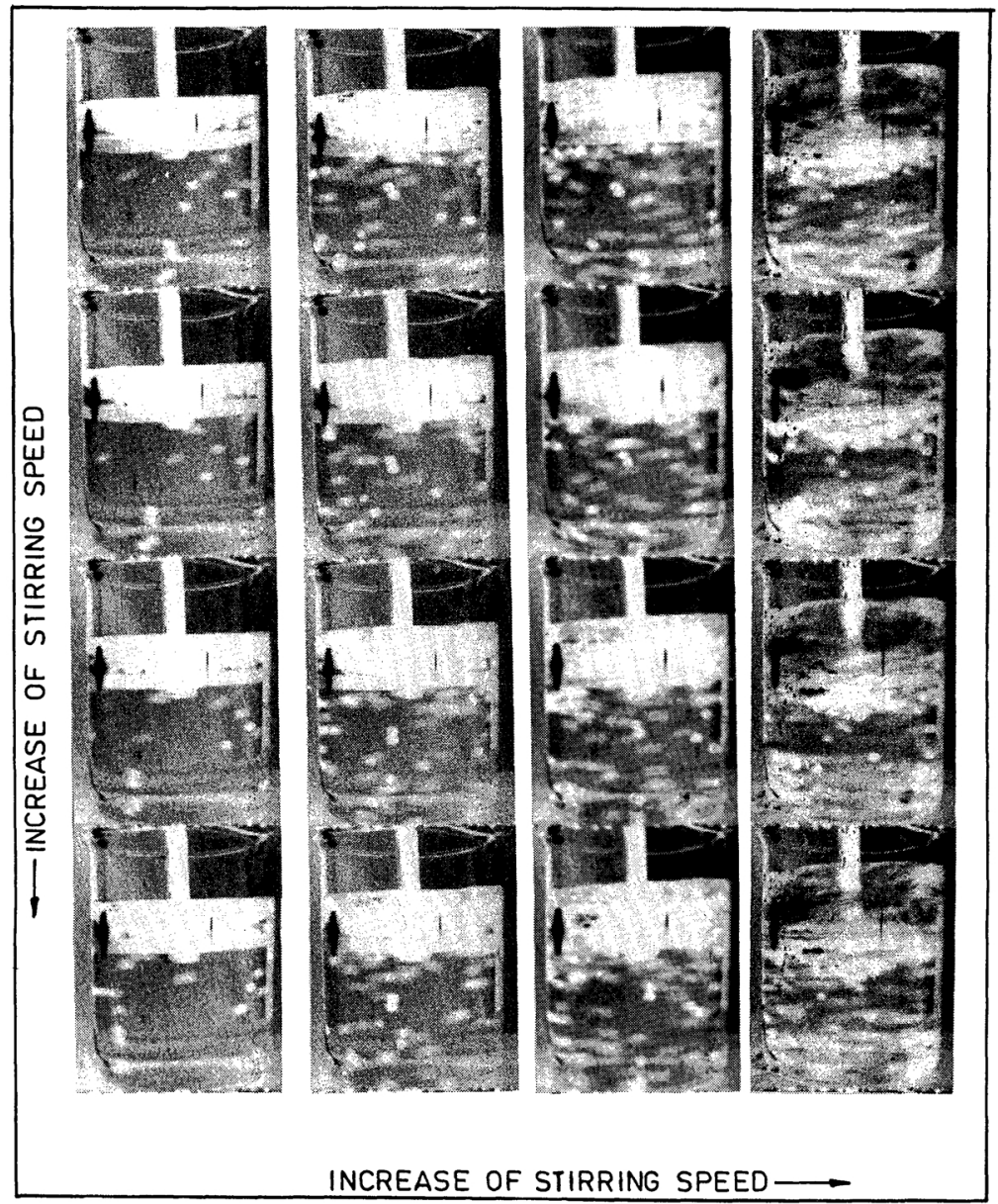

Fig. 3 Photographs showing the effect of stirring speed on the incorporation of plastic beads in water during the cold model experiment with an impeller of $d / D=0.66$.

relation between the size of the impeller and the speed of stirring at the beginning of exposure of the impeller surface is shown in Fig. 5. It has been observed that the exposure of the impeller starts at a higher speed of stirring for an impeller having a lower $d / D$ ratio.

During the experiments the beads have been visually observed to enter into water with an air envelope appearing as a bright layer over the beads. When the surface of the vortex has come down below the impeller, the air bubbles are accumulated at the bottom of the impeller retaining a substantial amount of beads along it.

\section{Discussion}

The cold model experiments have revealed the nature of the vortex and the importance of the exposure of the impeller vis-a-vis the retention of the plastic beads in water. The mechanism of the incorporation of the solid in the agitated liquid has been understood from the model experiments as shown in Figs. 2, 3 and 4 . In a concentrically agitated mixing process the height of the liquid surface comes down at the centre during stirring as the speed of the stirrer increases ${ }^{(9)(10)}$. Beyond a certain stirring speed a part of the stirrer becomes exposed and the particles floating on the liquid 


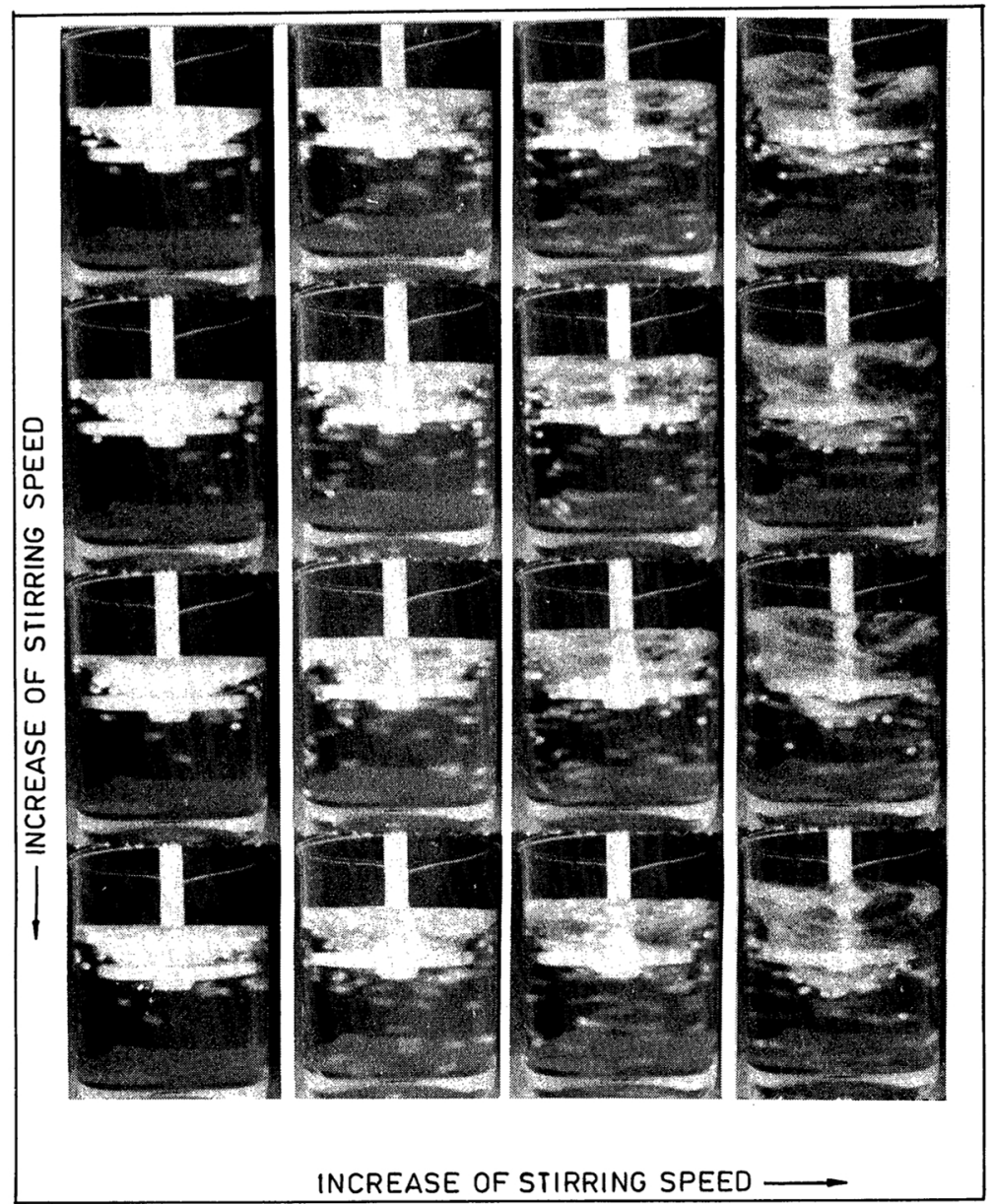

Fig. 4 Photographs showing the effect of stirring speed on the incorporation of plastic beads in water during the cold model experiment with an impeller of $d / D=0.48$.

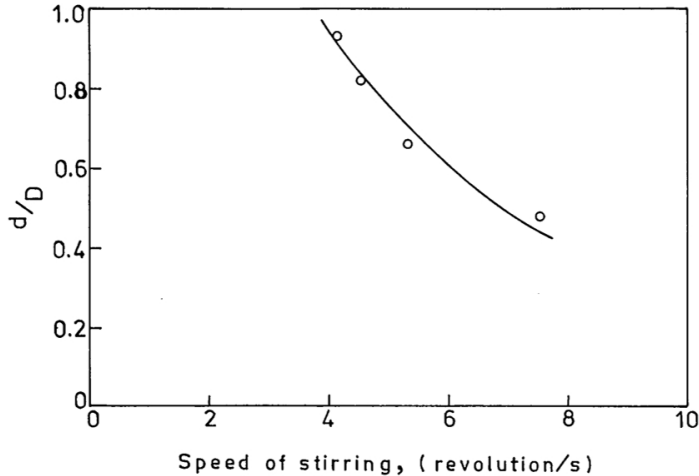

Fig. 5 The critical speed for incorporation at impellers with different $d / D$ ratios. surface are drawn towards the centre by the extended free vortex region and fall on the exposed rotating stirrer. These particles are scattered by the stirrer and thus gain an inertial centrifugal force enabling them to enter the liquid. Under this inertial force the particles sometimes even impinge on the wall of the container and gets distributed throughout the liquid under the influence of its turbulent flow.

The schematic diagram of the impeller (Fig. 1) shows that the impellers used in this investigation are having a central zone of radius, $r_{3}$, extending to the blades. With the increase in the stirring speed, when the radius of cylindrically rotating zone of liquid, $r_{c}$, exceeds the value of $r_{3}$ as presented schematically in Fig. 1, 
a large size bubble forms due to existence of a low pressure at the boundary of the forced vortex region. When $r_{\mathrm{c}}<r_{3}$ the low pressure does not cause suction of bubbles due to the existence of the continuous region of the stirrer. For a different design of stirrer like the turbine type, the bubble will be sucked through vortex at a low speed ${ }^{(9)(10)}$. Under this circumstance a considerable amount of air bubbles are sucked inside the liquid and these bubbles rise to the vortex region at the bottom of the impeller (Fig. 3). The beads already incorporated inside the water are carried eventually to the vortex below the impeller (Fig. 3) by the upward flow of the liquid from the lower region of the vessel towards it and thereby minimises the retention of beads in the matrix.

During stirring of water with a bigger impeller of $d / D=0.82$ the vortex has not been observed to come down below the impeller (Fig. 2 ) at any stirring speed used in this investigation because of a comparatively larger $r_{3}$. At a given stirring speed the Reynold's number, $R e$, is more in the case of an impeller of higher $d / D$ ratio as ${ }^{(9)}$

$$
R e=d^{2} n \rho^{\prime} / \eta
$$

and it results in a larger value of $r_{\mathrm{c}}$ as ${ }^{(9)}$

$$
\operatorname{Re} /\left(10^{3}+1.6 \operatorname{Re}\right)=r_{\mathrm{c}} / r_{1}
$$

Here, $n$ is the stirring speed (revol.s $\left.{ }^{-1}\right), \rho^{\prime}$ is the density of liquid, $\eta$ is the coefficient of viscosity of the liquid and $r_{1}$ is the radius of the impeller. Thus, at a given stirring speed the height of the bottom of the vortex surface from the bottom of the vessel, $Z_{0}$, has been estimated $^{(9)}$ by the following equation:

$$
\begin{aligned}
& \left(H-Z_{0}\right) / r_{2}^{2}\left(g / \omega^{2}\right) \\
& =y^{2}-y^{4}\{2.303 \log (1 / y)+0.75\},
\end{aligned}
$$

where $H$ is the height of the liquid at rest, $y=r_{\mathrm{c}} / r_{2} \leqslant 1, \omega$ is the angular velocity estimated as $2 n \pi, g$ is the accelaration due to gravity, and $r_{2}$ is the radius of the cylindrical vessel. Thus, at a given stirring speed the value of $Z_{0}$ will be lower in case of stirring with a bigger impeller than that estimated for a smaller impeller. So it can be inferred that during stirring with an impeller of higher $d / D$ ratio the impeller will be exposed at a lower sitrring speed than that for an impeller of lower size. As a result the incorporation of beads will take place at a lower speed of stirring where a bigger impeller is used as shown in Fig. 5. However, during stirring with an impeller of higher $d / D$ ratio the increase of $r_{\mathrm{c}}$ with the increase of the stirring speed reduces the volume of liquid under turbulence and, thus, adversely affects the mixing. Moreover, at higher stirring speeds the beads strike the vessel wall at a higher kinetic energy due to which the particles could not be deflected enough downwards to go into the region below the impeller and the beads come back again to the vortex after getting scattered by the container wall. Thus, at a higher speed of stirring with a stirrer of $d / D=0.82$ the incorporation is not favoured. It has also been observed that at high stirring speed the beads which are incorporated are accumulated at the bottom of the impeller as shown in Fig. 2 and reduces the retention of beads in the water matrix.

In the cold model experiment during stirring at a lower speed with the help of a smaller impeller $(d / D=0.48)$ the axial velocity of the discharge flow is low. It increases the thickness of the stagnant layer of the liquid adjacent to the vessel wall ${ }^{(9)}$. Thus, the beads incorporated inside the liquid have been observed to float out by the side of the vessel wall as shown in Fig. 4. Due to the lower $r_{3}$ of this impeller, $r_{c}$ exceeds the value of $r_{3}$ at a certain value of increasing stirring speed where the accumulation of air bubbles and particles occurs at the bottom of the impeller as discussed earlier. However, due to the small $r_{3}$ the bubbles become unstable by the accumulation of air and floats out above the impeller along with the accumulated beads. With a further increase in stirring speed a substantial part of the impeller surface becomes exposed and $r_{\mathrm{c}}$ becomes much larger than $r_{3}$. Under this circumstance the air bubbles accumulated at the bottom of the impeller comes out frequently with the growth of its size. This process along with the severity of turbulence disturbs the entire flow system. Thus, the beads already inside the liquid float out of the liquid through the side of the vessel wall.

The incidence of high porosity in foundry 
composites results also from the incorporation of air bubbles either as an envelope on particle or by direct suction. When negative pressure exist at the vortex the particle and air bubble both will enter the liquid under suction and thus the observed trend of higher porosity with greater incorporation of particle can be qualitatively explained.

\section{Conclusion}

The cold model experiment carried out by mixing plastic beads in water confirms that the exposure of the impeller surface significantly enhances the extent of incorporation of beads into agitated water, because the beads fall on the rotating surface of the impeller and enter the liquid after being scattered by the stirrer. The exposure of the impeller starts at a higher speed of stirring for an impeller of lower $d / D$ ratio. When the stirring speed is lower than its optimum level there is no transfer of beads into water due to non exposure of the impeller. The retention of beads inside the liquid is largely governed by the characteristics of the fluid motion. At a stirring speed higher than the op- timum one beads are rejected and sometimes, accumulates at the bottom of the impeller when the vortex comes down below it. During stirring the air bubbles enter inside the liquid by suction at the centre of the vortex and also as an envelope to the plastic beads.

\section{REFERENCES}

(1) R. Mehrabian, R. G. Rick and M. C. Flemings: Met. Trans., 5 (1974), 1899.

(2) R. Mehrabian, A. Sato and M. C. Flemings: The Light Metals, II (1975), 177.

(3) M. K. Surappa and P. K. Rohatgi: J. Mater. Sci., 16 (1981), 983.

(4) P. K. Ghosh, S. Ray and P. K. Rohatgi: Trans. JIM, 25 (1984), 440.

(5) B. F. Quigley, G. J. Abbaschian, R. Wunderlin and R. Mehrabian: Met. Trans., 13A (1982), 93.

(6) P. K. Ghosh, P. R. Prasad and S. Ray: Z. Metallk., 75 (1984), 934.

(7) P. K. Ghosh and S. Ray: J. Mater. Sci., 21 (1986), 1667.

(8) P. K. Ghosh and S. Ray: J. Mater. Sci., 22 (1987), 4077.

(9) S. Nagata: Mixing Principles and Applications, J̣ohn Wiley and Sons, New York, (1975), 1.

(10) P. K. Ghosh and S. Ray: in press, Indian J. Tech., Silver jublee special issue, (1988). 